\title{
Vibrational Frequencies and Raman Radial Breathing Modes of Multi-Walled Carbon Nanotubes Based on Continuum Mechanics
}

\author{
Toshiaki Natsuki ${ }^{1}$, Gan Jet Hong Melvin ${ }^{2} \&$ Qing-Qing Ni ${ }^{3}$ \\ ${ }^{1}$ Department of Functional Machinery \& Mechanics, Shinshu University, 3-15-1 Tokida, Ueda, Nagano, Japan \\ ${ }^{2}$ Interdisciplinary Graduate School of Science and Technology, Shinshu University, 3-15-1 Tokida, Ueda, \\ Nagano Japan \\ ${ }^{3}$ Department of Functional Machinery \& Mechanics, Shinshu University, 3-15-1 Tokida, Ueda, Nagano, Japan \\ Correspondence: Toshiaki Natsuki, Department of Functional Machinery \& Mechanics, Shinshu University, \\ 3-15-1 Tokida, Ueda, Nagano 386-8567, Japan. Fax: 81-268-215-482. E-mail: natsuki@shinshu-u.ac.jp
}

Received: May 23, 2013 Accepted: June 23, 2013 Online Published: July 12, 2013

doi:10.5539/jmsr.v2n4p1 URL: http://dx.doi.org/10.5539/jmsr.v2n4p1

\begin{abstract}
Carbon nanotube structures correspond to the vibrational frequencies and modes of CNTs. In this study, the vibrational frequencies and the Raman RBM (radial breathing mode) of multi-walled carbon nanotubes (MWCNTs), and carbon nanotubes (CNTs) embedded in elastic medium are simulated based on a continuum mechanics. According to this the theoretical analysis, we can understand how the vibrational modes of CNTs effect the RBM frequency shift. It is found that the vibrational frequency of RBM is complicated due to the van der Waals (vdW) interaction between layers of MWCNTs. The highest RBM frequency of MWCNTs depends mostly on the diameter of the innermost layer, being insensitive to the layer numbers. The analytical approximation offers a useful method for determining precisely the diameter of the MWCNTs.
\end{abstract}

Keywords: multi-walled carbon nanotubes, radial breathing modes, Raman spectroscopy, simulation

\section{Introduction}

The vibration behavior of carbon nanotubes (CNTs) has been extensively investigated due to their importance in nano-electro-mechanical systems (NEMS) and nanosensor application. Raman spectroscopy is a technique widely used to study the vibrational modes of CNTs and characterize their structures (Binnig \& Quate, 1986; Jorio et al., 2003; Dresselhaus et al., 2005; Aydin \& Akins, 2010). The Raman spectrum analysis usually focuses on three specific frequencies, which are low-frequency radial breathing mode (RBM) and other higher-frequency modes such as D- and G-bands. The D-band, known as the disorder or defect mode, originates from edge configurations in graphene where the planar sheet configuration is disrupted. The G-band is the primary mode in graphene and it provides a good representation of $\mathrm{sp}^{2}$ bonded carbon. Therefore, the ratio of D-band to G-band is usually used for evaluating the quality or purity of CNTs. In the Raman analysis of CNTs, a low-frequency peak with less than $300 \mathrm{~cm}^{-1}$ are assigned to the RBM, which is very sensitive to the nanotube diameters (Dresselhaus et al., 2005).

The molecular structure of single-walled carbon nanotubes (SWCNTs) can be considered as graphene sheet of carbon atoms rolled into seamless hollow cylinder, and multi-walled carbon nanotubes (MWCNTs) are regarded as several co-axially arranged SWCNTs with varying diameter. Although the D- and G-bands are found in graphite, the RBM is specific to CNTs and is representative of the isotropic radial expansion of CNTs. Therefore, the Raman RBM is an important parameter for determining the distribution of diameters for CNT samples. The RBM corresponds to the coherent vibration of the carbon atoms in the radial direction, and the RBM frequency of SWCNTs has been proved to be inversely proportional to the tube diameter (Popov et al., 1999; Venkateswaran et al., 2003). Venkateswaran et al. (2003) have studied the pressure-dependance of RBM frequency. They were able to fit the experimental data with a functional form obtained from a simple elastic model.

Generally, the molecular dynamics (MD) simulation can be used to investigate the RBM frequencies (Lawler et al., 2003; Kurti et al., 1998) and the mechanical properties of CNTs (Mylvaganam \& Zhang, 2004; Qian et al., 2002; Yakobson, 1998; Zhang et al., 1998; Srivastava et al., 1998), but the MD method is time consuming 
computation and demands an available computational power of hardware, especially for larger atom numbers. A simulation method that reduces the computational cost is a challenging task for studying larger atomic systems in engineering calculations. At present, the dynamical properties of CNTs have been effectively studied based on the continuum mechanics due to relatively less calculation time and good prediction (Agrawal et al., 2006; Natsuki et al., 2004; Hu et al., 2008; Wang et al., 2006; Natsuki et al., 2008). At present, low-frequency phonons in CNTs have been calculated using the continuum approximation (Chico et al., 2006; Mahan, 2002). The continuum model allowed consideration of an arbitrary wall thickness for the nanotubes. Chico et al. (2006) analyzed the effect of the nanotube wall thickness on the eigenfrequency of CNTs based on a thin-shell approximation. They reported that several modes did have a strong dependence on the parameter. Mahan (2002) obtained an analytical formula for the Raman frequencies and for the velocities of acoustical modes. The general formulas can be applied to the breathing modes of SWCNTs, which is in good agreement with the experimental results. Based on MD simulation and non-local continuum model, Shi et al. showed 2:1 internal resonance between the RBM and the circumferential flexural mode (CFM) in SWCNTs (Shi et al., 2009). They observed that the change of the transformation time from RBM to CFM due to the variation of the perturbation velocity field. Although some studies of Raman RBM of double- or multi-walled CNTs also have been carried out using continuum mechanics (Wu et al., 2007; Wang \& Ru, 2005; Han \& Goddard, 2009; Popov \& Henrard, 2002) these analytical models were discussed theoretically and there was a lack of experimental investigation. Popov and Henrard (2002) studied the breathinglike phonon modes of MWCNTs using a valence force field model. The predicted intensity of breathinglike modes was compared to available low-frequency experimental data. It was shown that their predictions correspond well the experimental resonant Raman spectrum of two-layer tubes.

In this work, we present an analytical solution for Raman RBM frequency of MWCNTs using the circular ring mode. The effects of CNT diameters and layer numbers on the RBM frequency are discussed in detail using the present approach. The analytical approximation can offer an estimation for determining the diameter and layer number of the MWCNTs. Moreover, the elastic medium dependency of embedded matrix on the RBM frequency of double-walled carbon nanotubes (DWCNTs) is also simulated and investigated. The calculated results were compared to the experimental observation, showing a good agreement in the frequency shifts.

\section{Theoretical Approach}

\subsection{Governing Equations}

Love's shell theory is proposed as the governing differential equations of vibration of CNTs (Soedel, 2006). For a circular ring, where $v$ and $w$ are the deflections in the circumferential $\theta$ and the radial directions, respectively, the vibration equations can be given by

$$
\begin{aligned}
& \frac{D}{R^{4}}\left(\frac{\partial^{2} v}{\partial \theta^{2}}-\frac{\partial^{3} w}{\partial \theta^{3}}\right)+\frac{K}{R^{2}}\left(\frac{\partial^{2} v}{\partial \theta^{2}}+\frac{\partial w}{\partial \theta}\right)=\rho h \frac{\partial^{2} v}{\partial t^{2}} \\
& \frac{D}{R^{4}}\left(\frac{\partial^{3} v}{\partial \theta^{3}}-\frac{\partial^{4} w}{\partial \theta^{4}}\right)-\frac{K}{R^{2}}\left(\frac{\partial v}{\partial \theta}+w\right)+p=\rho h \frac{\partial^{2} w}{\partial t^{2}}
\end{aligned}
$$

where $R$ is nanotube radius, $p$ is the distributed radial pressure acting on the nanotube per unit axial length, $\rho$ is the mass density, and $t$ is time.

The CNTs can be regarded as rolled graphitic layers and to have a finite wall thickness of a graphene sheet, which are much less than the diameter of CNTs. Therefore, the bending stiffness $(D)$ and membrane stiffness $(K)$ of CNTs can be given by

$$
D=\frac{E h^{3}}{12\left(1-\mu^{2}\right)}, K=\frac{E h}{1-\mu}
$$

where $E$ and $\mu$ is the elastic modulus and Poisson's ratio of the graphene sheet, respectively.

Eliminating $v$ form Equations (1) and (2), we have

$$
L_{A} w+L_{B} p=0
$$

where $L_{A}$ and $L_{B}$ is the differential operators, given by

$$
L_{A}=(\rho h)^{2} \frac{\partial^{4}}{\partial t^{4}}+\rho h \frac{\partial^{2}}{\partial t^{2}}\left\{\frac{D}{R^{4}} \frac{\partial^{4}}{\partial \theta^{4}}-\left(\frac{D}{R^{4}}+\frac{K}{R^{2}}\right) \frac{\partial^{2}}{\partial \theta^{2}}+\frac{K}{R^{2}}\right\}-\left(\frac{D K}{R^{6}}\right) \frac{\partial^{2}}{\partial \theta^{2}}\left(\frac{\partial^{2}}{\partial \theta^{2}}+1\right)^{2}
$$




$$
L_{B}=\left(\frac{D}{R^{4}}+\frac{K}{R^{2}}\right) \frac{\partial^{2}}{\partial \theta^{2}}-\rho h \frac{\partial^{2}}{\partial t^{2}}
$$

For the DWCNTs, the coupled equations of the pressure $p$ in Equation (4) caused by the van der Waals (vdW) interaction between the inner and outer nanotubes can be given by

$$
\begin{aligned}
& p_{1}=c\left(w_{2}-w_{1}\right) \\
& p_{2}=c\left(w_{1}-w_{2}\right)
\end{aligned}
$$

which the subscripts 1 and 2 from Equation (7) and Equation (8) denote the inner and outer nanotube, respectively. $c$ is the $\mathrm{vdW}$ interaction coefficient between the inner and outer nanotubes of DWCNTs, which can be obtained from Lennard-Jones potential, given as (He et al., 2005)

$$
c=\frac{\pi \varepsilon R_{1} R_{2} \sigma^{6}}{a^{4}}\left[\frac{1001 \sigma^{6}}{3} H^{13}-\frac{1120}{9} H^{7}\right]
$$

where

$$
H^{m}=\left(R_{1}+R_{2}\right)^{-m} \int_{0}^{\pi / 2} \frac{d \theta}{\left(1-\varphi \cos ^{2} \theta\right)^{m / 2}} \quad(m=7,13)
$$

and

$$
\varphi=\frac{4 R_{1} R_{2}}{\left(R_{1}+R_{2}\right)^{2}}
$$

where $a$ is the carbon-carbon bond length $(0.142 \mathrm{~nm}), R_{1}$ and $R_{2}$ are the inner and outer radii of the DWCNTs, and $\sigma$ and $\varepsilon$ are the vdW radius and the well depth of the Lennard-Jones potential, respectively. The vdW parameters in the Lennard-Jones potential are taken as $\sigma=0.34 \mathrm{~nm}$ and $\varepsilon=2.967 \mathrm{meV}$ (Saito et al., 2007).

Substuting Equations (7) and (8) into Equation (4), the coupled governing equations of the vibration in DWCNTs are written as

$$
\begin{aligned}
& L_{A}^{1} w_{1}+L_{B}^{1}\left\{c\left(w_{2}-w_{1}\right)\right\}=0 \\
& L_{A}^{2} w_{2}+L_{B}^{2}\left\{c\left(w_{1}-w_{2}\right)\right\}=0
\end{aligned}
$$

\subsection{The Solution of Governing Equations}

The motion governing equations constituted by Equations (12) and (13) are high-order linear differential equations. The harmonic solution of the equations can be expressed as

$$
w_{j}=A_{j} \cos (n \theta+\omega t) j=1,2
$$

where $A_{j},(j=1,2)$ is the amplitude of vibration in the inner and outer tubes. $\omega$ is the vibration frequency. $n$ is the circumferential wave number, which characterizes the vibration mode shapes of nanotubes.

Substituting Equation (14) into Equations (12) and (13), we obtain the coupled equation, given in matrix form as

$$
\left[\begin{array}{cc}
(\rho h)^{2} \omega^{4}-\frac{\left(n^{2}+1\right) \rho h \omega^{2}}{R_{1}^{2}}\left(\frac{n^{2} D}{R_{1}^{2}}+K\right)-c \rho h \omega^{2}+c\left(\frac{D}{R_{1}^{4}}+\frac{K}{R_{1}^{2}}\right) n^{2} & -c\left(\frac{D}{R_{1}^{4}}+\frac{K}{R_{1}^{2}}\right) n^{2}+c \rho h \omega^{2} \\
-c\left(\frac{D}{R_{2}^{4}}+\frac{K}{R_{2}^{2}}\right) n^{2}+c \rho h \omega^{2} & (\rho h)^{2} \omega^{4}-\frac{\left(n^{2}+1\right) \rho h \omega^{2}}{R_{2}^{2}}\left(\frac{n^{2} D}{R_{2}^{2}}+K\right)-c \rho h \omega^{2}+c\left(\frac{D}{R_{2}^{4}}+\frac{K}{R_{2}^{2}}\right) n^{2}
\end{array}\right]\left(\begin{array}{l}
A_{1} \\
A_{2}
\end{array}\right)=0
$$

The vibration frequency of CNTs can be determined by the nontrivial solution in Equation (15). The vibration amplitude ratio of the inner to the outer tubes is

$$
\frac{A_{1}}{A_{2}}=\frac{-c\left(\frac{D}{R_{1}^{4}}+\frac{K}{R_{1}^{2}}\right) n^{2}+c \rho h \omega^{2}}{(\rho h)^{2} \omega^{4}-\frac{\left(n^{2}+1\right) \rho h \omega^{2}}{R_{1}^{2}}\left(\frac{n^{2} D}{R_{1}^{2}}+K\right)-c \rho h \omega^{2}+c\left(\frac{D}{R_{1}^{4}}+\frac{K}{R_{1}^{2}}\right) n^{2}}
$$


For SWCNTs, the vibration frequencies of the transverse deflection $\left(\omega_{n 1}\right)$ and the circumferential deflection $\left(\omega_{n 2}\right)$ can be given by simple formulas from Equation (15)

$$
\left.\begin{array}{c}
\omega_{n 1} \\
\omega_{n 2}
\end{array}\right\}=\left\{\frac{K_{1}}{2}\left(1 \mp \sqrt{1-\frac{4 K_{2}}{K_{1}^{2}}}\right)\right\}^{\frac{1}{2}}
$$

where

$$
K_{1}=\frac{n^{2}+1}{\rho h R^{2}}\left(\frac{n^{2} D}{R^{2}}+K\right), \quad K_{2}=\frac{n^{2}\left(n^{2}-1\right)^{2}}{(\rho h)^{2} R^{6}} D K
$$

The mode shape of $n=0$ is called the radial breathing mode (RBM). At $n=1$, a bending vibration does not exist. We think of the ring as being displaced in a rigid-body motion. Two positive roots in the nontrivial solution correspond to the vibration frequencies in the transverse and circumferential directions. The transverse deflection of CNTs starts from $n=2$.

At $n=0$, we can obtain the vibrational frequency of RBM from Equations (17) and (18)

$$
\omega=\frac{K}{\rho h R^{2}}
$$

\subsection{Vibration Frequency of Dwctns Embedded in Matrix}

For the DWCNTs embedded within an elastic medium, a Winkler spring model has been used to deal with the interface between CNTs and matrix. The pressure $p$ per unit axial length, acting on the outer nanotube due to the surrounding elastic medium, can be described as

$$
p=-k_{0} w_{N}
$$

where the negative sign indicates that the pressure $p$ is opposite to the deflection of the nanotube. $w_{N}$ is the deflection of the outermost tube. $k_{0}$ is a spring constant determined by the material constant of the elastic medium.

Consider that the outer tube of DWCNTs is subjected to the pressures by the elastic medium and the inner tube, therefore, Equations (12) and (13) can be rewritten as

$$
\begin{gathered}
L_{A}^{1} w_{1}+L_{B}^{1}\left\{c\left(w_{2}-w_{1}\right)\right\}=0 \\
L_{A}^{2} w_{2}+L_{B}^{2}\left\{c w_{1}-\left(c+k_{0}\right) w_{2}\right\}=0
\end{gathered}
$$

According to the Whitney-Riley model, the spring constant $k_{0}$ is given by Yang and Wang (2007)

$$
k_{0}=p\left(w_{r=R_{N}}\right) \frac{E_{m}}{1+\mu_{m}} \frac{1}{R_{N}}
$$

where $E_{m}$ and $\mu_{m}$ are the modulus and the Poisson's ratio of the elastic medium, respectively. $R_{N}$ is the outermost radius of DWCNTs.

\subsection{Raman RBM Vibration of MWCNTs}

The RBM frequency of MWCNTs occurs in the vibration mode $n=0$. Applying Equation (4) to each layer (Radius $R_{j}, j=1, \cdots, N$ ) of all concentric nanotubes of an individual MWCNT, the N-coupled governing equations for MWCNTs are expressed as

$$
\begin{gathered}
-\rho h \omega^{2} A_{1}+\frac{K}{R_{1}^{2}} A_{1}-c_{1}\left(A_{2}-A_{1}\right)=0 \\
-\rho h \omega^{2} A_{2}+\frac{K}{R_{2}^{2}} A_{2}-c_{1}\left(A_{1}-A_{2}\right)-c_{2}\left(A_{3}-A_{2}\right)=0 \\
-\rho h \omega^{2} A_{N}+\frac{K}{R_{N}^{2}} A_{N}-c_{N-1}\left(A_{N-1}-A_{N}\right)=0
\end{gathered}
$$

where $c_{j},(j=1, \cdots, N)$ are the vdW interaction coefficient between the $j$ th and $(j+1)$ th layers. $A_{j}(j=1, \cdots, N)$ are the vibration amplitudes of each nanotube. 
A nontrivial solution of Equation (24) determines the RBM frequencies of MWCNTs and the relative amplitude ratios $A_{j} / A_{N}, j=1, \cdots, N$.

\section{Results and Discussion}

In this simulation, the material constants of CNTs are the elastic modulus of $1 \mathrm{TPa}$, the mass density of 2.27 $\mathrm{g} / \mathrm{cm}^{3}$ and the Poisson's ratio of 0.16 . The effective layer thickness of one layer tube was taken to be that of graphene sheet with $0.34 \mathrm{~nm}$.

The RBM mode is defined by the circumferential wave number of $n=0$, which indicate that the vibrational frequency of RBM mode has the lowest value. For the circumferential wave number $n=0$, the RBM frequency of SWCNTs is shown in Figure 1 as a function of nanotube diameter. The RBM frequency decreases with increasing diameter. To examine the precision of predicted results, the data of the experimental results by Raman spectroscopy (Liu et al., 2001; Fantini et al., 2004) and the MD simulation (Popov \& Henrard, 2002) are plotted in the Figure 1. The prediction results quite agree with experimental data of Raman measurement and the classical MD simulation.

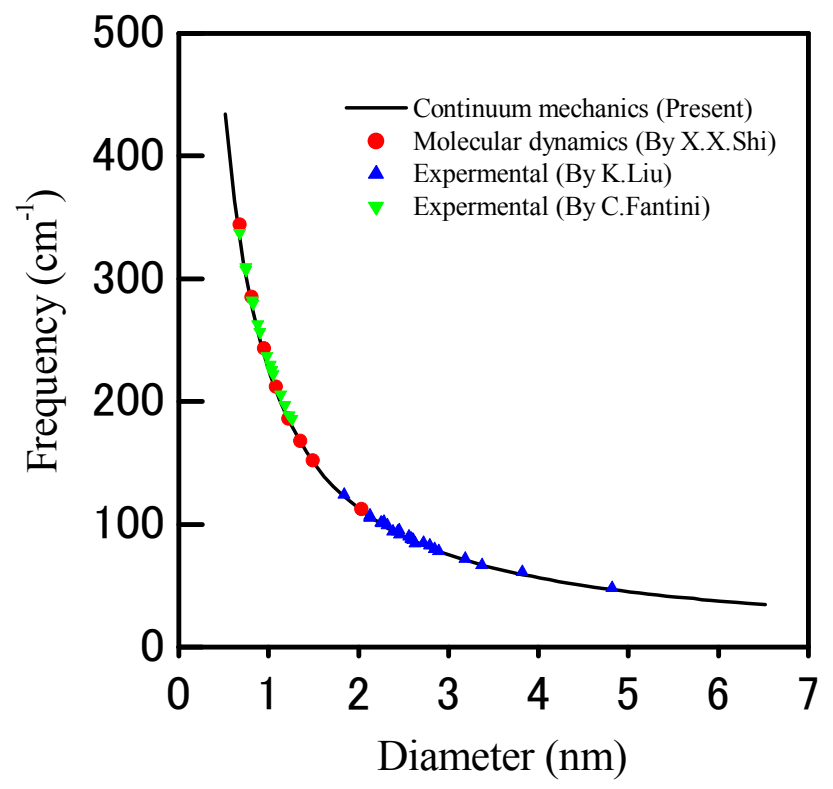

Figure 1. Relationship between RBM frequency and tube diameter for SWCNTs

In Figure 2, RBM frequencies of both SWCNTs and DWCNTs are shown as a function of nanotube diameter (the inner layer diameter for DWCNTs). The DWCNTs exist in two RBMs, and the RBM frequency of SWCNTs is found to be in between to those two RBMs of DWCNTs. The frequency curve of SWCNTs goes asymptotically to the DWCNT curves. In small tube diameter, the RBM frequency of SWCNTs is close to the high frequency of DWCNTs. As the tube diameter increased, however, the value of frequency tends to that of the low frequency of DWCNTs. The existences of two RBM frequencies of DWCNTs are caused by the vibration displacement between the inner and outer tubes that has the same or the opposite phase. The simulation results indicate the RBM vibrations of in-phase and anti-phase modes corresponding to the low and high frequencies, respectively. The Raman scattering from DWCNTs were experimentally measured by Kuzmany et al. (2008). The upper spectrum of DWCNT was more or less a replica of the high frequency, which were around 315 and $345 \mathrm{~cm}^{-1}$ for the inner-shell $(6,5)$ and $(6,4)$ tubes, respectively. According to the calculation, we predict that the theoretical values are the 314 and $341 \mathrm{~cm}^{-1}$, respectively. For low-frequency of DWCNT with in-phase mode, the observed Raman shifts were 164 and $182 \mathrm{~cm}^{-1}$ for the inner-shell $(6,5)$ and $(6,4)$ tubes, and the simulation values are 174 and $181 \mathrm{~cm}^{-1}$ respectively. There is good agreement between the measured and predicted results, and the relative errors are less than $6 \%$. Figure 3 shows the vibration amplitude ratio as a function of CNT diameter. The negative amplitude ratio shown in Figure 3 indicates the opposite vibration directions of the outer and inner tubes. The vibration of anti-phase mode causes has higher frequency than that of in-phase mode. It is seen that 
the vibration amplitude ratio is near 1.0 or -1.0 , which means that the inner and outer tubes have the same vibration amplitude.

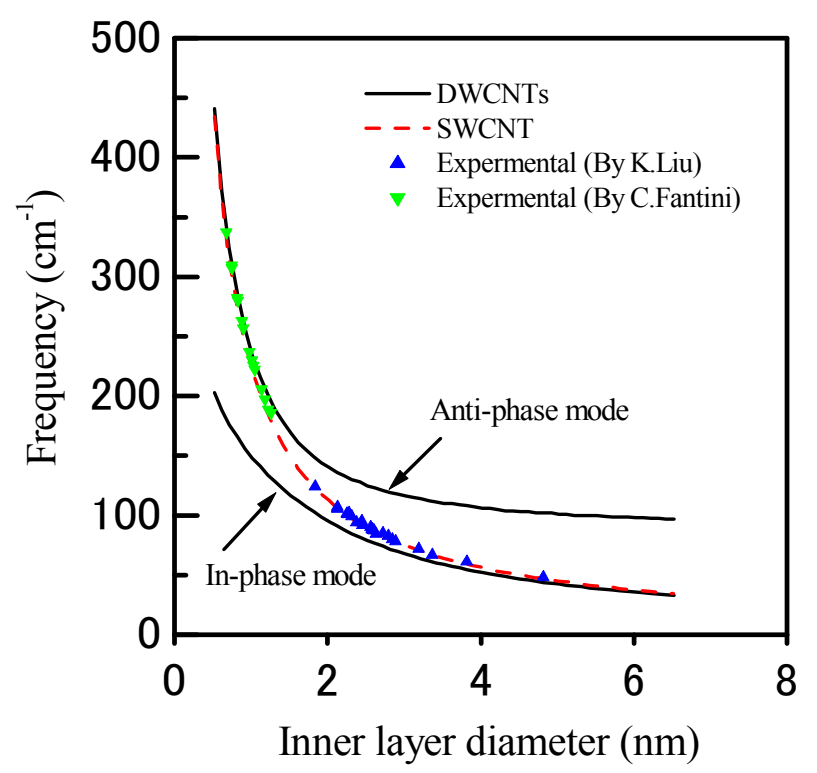

Figure 2. Influence of DWCNTs on the RBM frequency as a function of the inner layer diameter

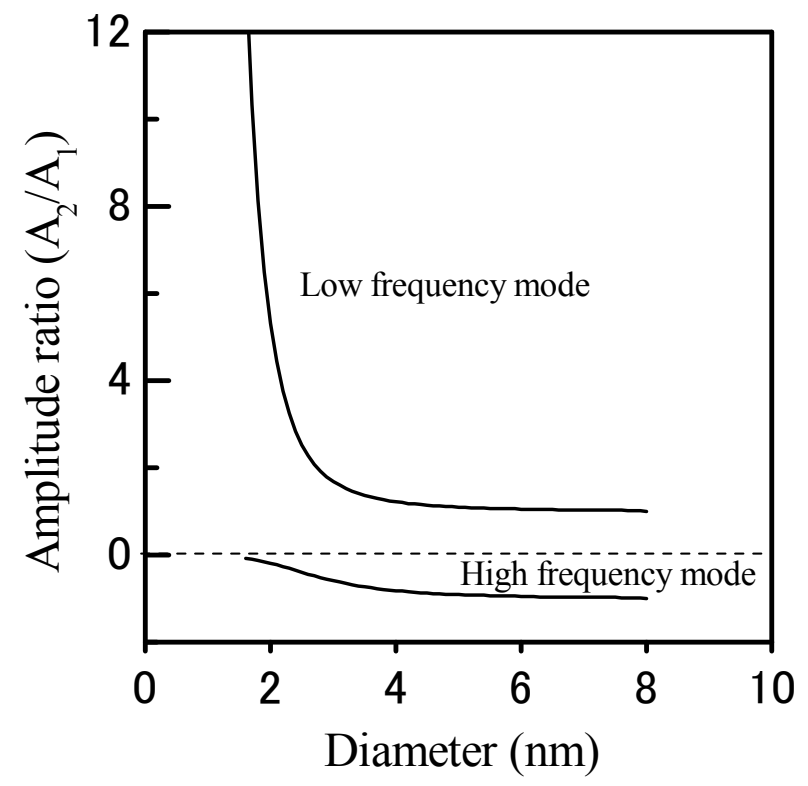

Figure 3. Influence of the tube diameter on amplitude ratio of the outer to inner tubes

RBM frequencies of triple-walled carbon nanotubes (TWCNTs) were also calculated. Figure 4 shows the relationship between RBM frequency and the inner diameter, and a schematic of configuration with different models. For the three-layer tubes, the opposite vibration direction between the adjacent tubes indicates the anti-mode, responding to the highest frequency (A), as shown in Figure 4. The in-phase mode, characterized by identical vibration directions, has the lowest frequency $(\mathrm{C})$. The mixed mode for three-layer tubes exhibits an intermediate frequency (B). The vibration amplitude ratios, corresponding to the above three modes, are shown in Figure 5 as a function of tube diameter. It is found that the amplitude ratios tend to constant values with increasing diameter. For larger diameter TWCNTs with the in-phase mode, the vibration amplitudes are almost same for each layer of three concentric tubes because the amplitude ratios are near 1.0. For the mixed-phase mode, the vibration direction of two innermost tubes is consistent but opposite to the two outmost tubes. The 
ratio of the innermost to the outermost tubes $A_{1} / A_{3}$ is near -1.0. In high frequency vibration with the anti-phase mode, the amplitude ratios, $A_{1} / A_{2} / A_{3}$, are obtained to be $1.0 /-1.87 / 0.88$ for the inner diameter $6.0 \mathrm{~nm}$.
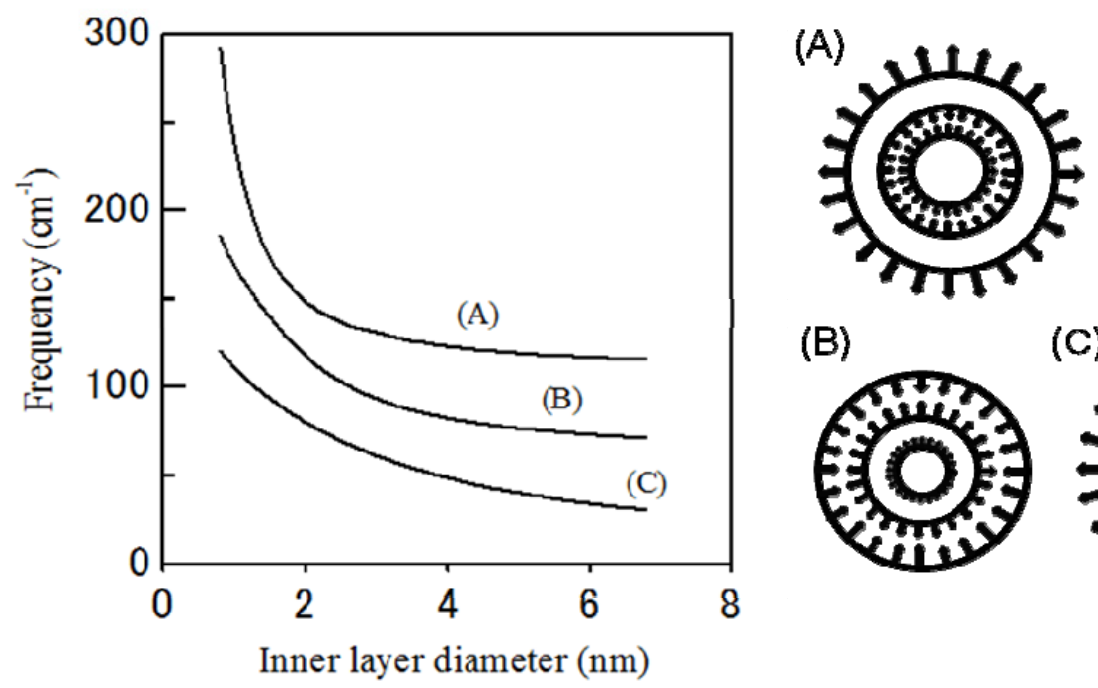

(B)
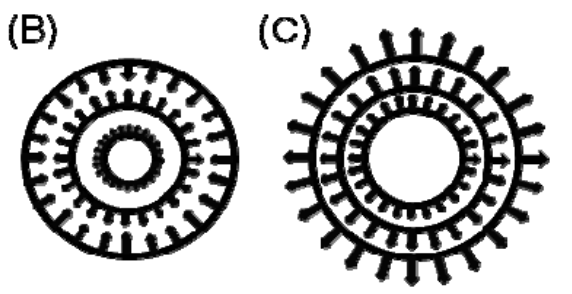

Figure 4. Relationship between RBM frequency of TWCNTs and the inner tube diameter
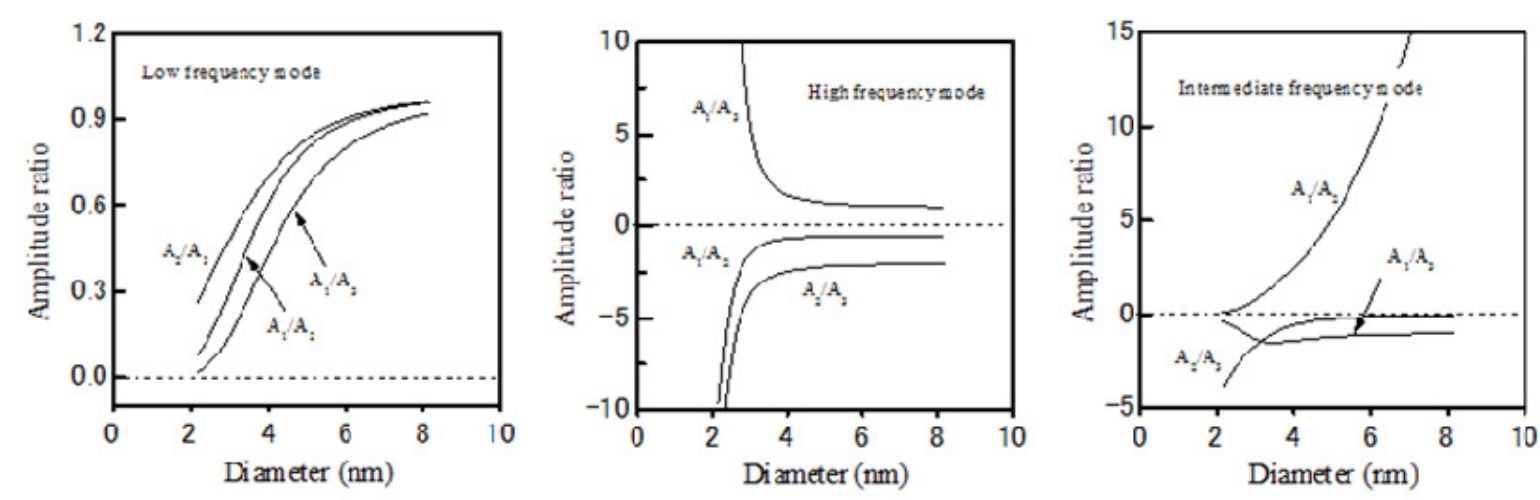

Figure 5. Amplitude ratio of the inner to outer tubes for TWCNTs as a function of tube diameter

Figure 6 shows the layer number dependence on the RBM frequency of MWCNTs, in which the innermost radius is fixed at $1.0 \mathrm{~nm}$. The highest frequency of MWCNT corresponds to the anti-phase mode, and tends to a constant value of about $150 \mathrm{~cm}^{-1}$ at more than four layers. This means that the highest vibration frequency of MWCNT is dominated by the innermost diameter. It is found that the lowest frequency, corresponding to the in-phase mode, decreases with increasing number of layers. The mixed phase modes of in-phase and anti-phase constitute many intermediate frequencies. The predicted results agree well with those of Popov and Henrard (2002). Figure 7 shows a comparison of Raman shift between the theoretical values and experimental measurement for MWCNTs with fixed innermost diameter of $1.2 \mathrm{~nm}$ (Benoit et al., 2004). For the five-layer CNTs, the relative error of each peak is within about $5 \%$. The highest frequency holds a constant frequency of about $198 \mathrm{~cm}^{-1}$ from 5 walls to 50 wall CNTs, which marches the calculated value. It is also proved that the lowest frequency decreases with increasing layer numbers. 


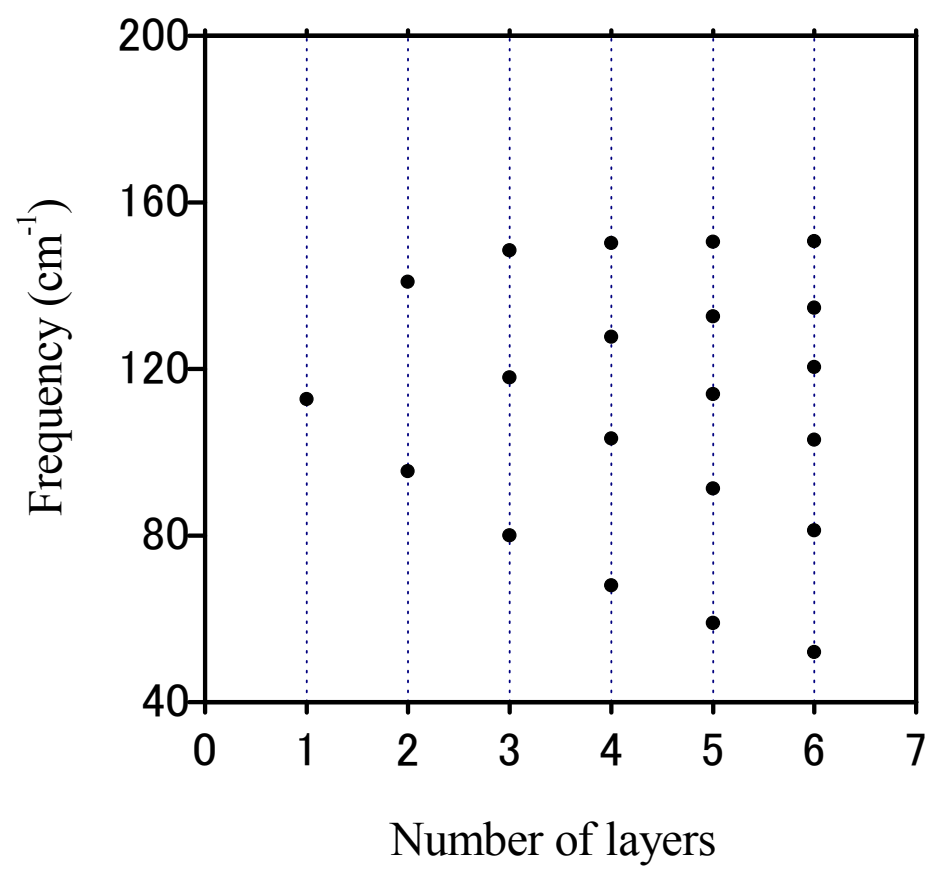

Figure 6. Dependence of layer numbers on the RBM frequency of MWCNTs

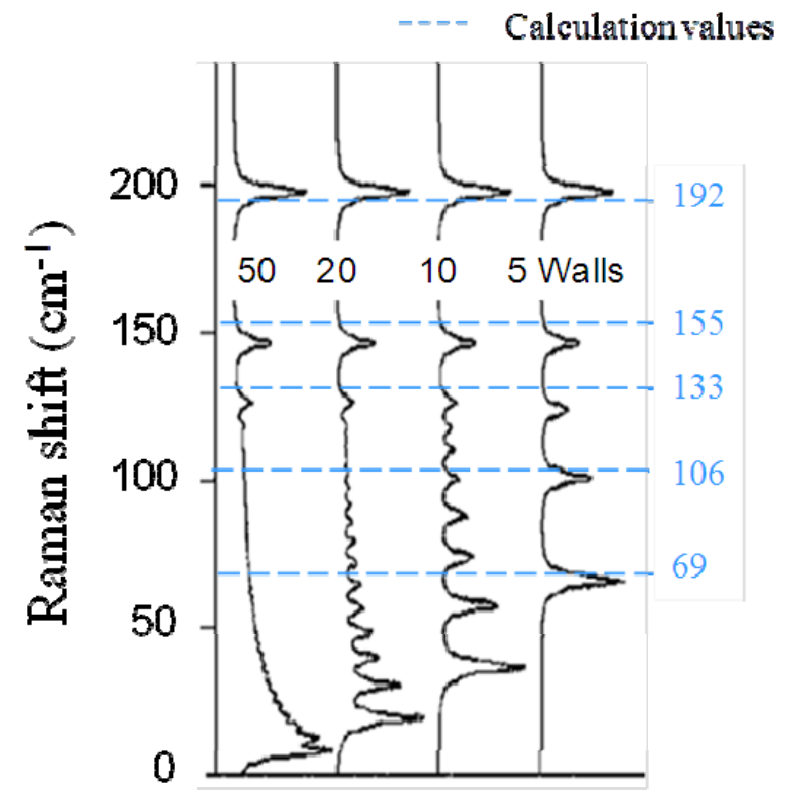

Figure 7. Comparison between the calculated values and the experimental measurement for MWCNTs

The RBM frequencies of embedded DWCNT are shown in Figure 8 as a function of tube diameter. Compared to higher RBM frequency, the lower frequency has more difference between the pristine and embedded DWCNTs. The Raman shift tends to increase when CNTs are embedded to elastic medium. Hasan et al. (2009) have used Raman spectroscopy to confirm the presence of SWCNTs within composites. The Raman shift predicted by the theoretical approach shows a correspondence with the Raman measurement results. 


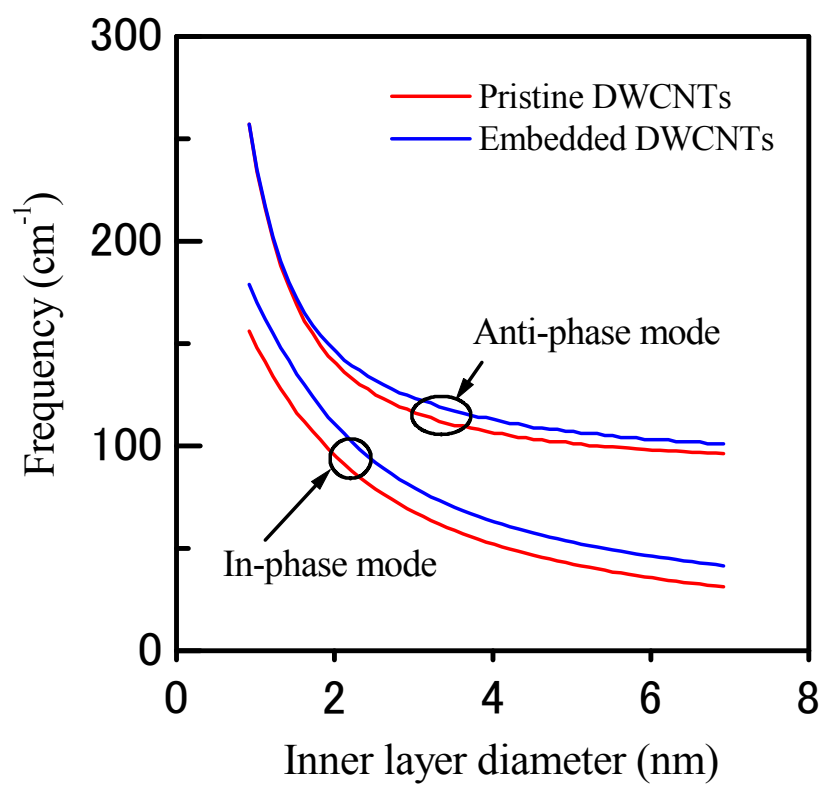

Figure 8. Influence of medium around CNT on the RBM frequency as a function of tube diamater

\section{Conclusion}

We present an analytical approach for the RBM of MWCNTs considered to be coupled together through the vdW interaction between tube layers. The Raman RBM of MWCNTs are simulated based on a continuum mechanics, and the effects of layer number of CNTs, embedded medium and the diameter on the RBM frequency are investigated in detail. The main results show as follows:

(1) The RBM frequencies of MWCNTs predicted by the present continuum mechanics are in good agreement with those of MD method and the experiment measures.

(2) The RBM frequencies of CNTs decrease with increasing diameter. The in-phase mode of MWCNTs corresponds to the lowest frequency, and the vibration amplitudes at each layer are stable/unchanged for larger diameter. The anti-phase mode has the highest vibration frequency.

(3) For the embedded DWCNTs, the Raman frequency shift tends to increase compared to the pristine DWCNTs. The result shows an agreement with Raman's measurement observation. The Raman frequency shift from the embedded to pristine DWCNTs has more difference in low vibrational mode.

\section{Acknowledgments}

This work was supported by Grants for Excellent Graduate Schools by the Ministry of Education, Culture, Sports, Science and Technology, Japan.

\section{References}

Agrawal, P. M., Sudalayandi, B. S., Raff, L. M., \& Komanduri, R. (2006). A comparison of different methods of Young's modulus determination for single-wall carbon nanotubes (SWCNT) using molecular dynamics $\begin{array}{lllll}\text { (MD) } & \text { simulations. } & \text { Computational } & \text { Materials } & \text { Science, }\end{array}$ http://dx.doi.org/10.1016/j.commatsci.2006.02.011

Aydin, M., \& Akins, D. L. (2010). Calculated dependence of vibrational band frequencies of single-walled and double-walled carbon nanotubes on diameter. Vibrational Spectroscopy, 53, 163-172. http://dx.doi.org/10.1016/j.vibspec.2010.02.001

Benoit, J. M., Buisson, J. P., Chauvet, O., Godon, C., \& Lefrant, S. (2002). Low-frequency Raman studies of multiwalled carbon nanotubes: experiments and theory. Physical Review B, 66, 073417-1-4. http://dx.doi.org/10.1103/PhysRevB.66.073417

Binnig, G., \& Quate, C. F. (1986). Atomic Force Microscope. Physical Review Letters, 56, 930-933. 
Chico, L., Álvarez, R. P., \& Cabrillo, C. (2006). Low-frequency phonons in carbon nanotubes: A continuum approach. Physical Review B, 73, 075425-1-7. http://dx.doi.org/10.1103/PhysRevB.73.075425

Dresselhaus, M. S., Dresselhaus, G., Saito, R., \& Jorio, A. (2005). Raman spectroscopy of carbon nanotubes. Physics Reports, 409, 47-99. http://dx.doi.org/10.1016/j.physrep.2004.10.006

Fantini, C., Jorio, A., Souza, M., Strano, M. S., Dresselhaus, M. S., \& Primenta, M. A. (2004). Optical transition energies for carbon nanotubes from resonant Raman spectroscopy: environment and temperature effects. Physical Review Letters, 93, 147406-1-4. http://dx.doi.org/10.1103/PhysRevLett.93.147406

Jorio, A, Piment, M. A., Filho, A. G. S., Saito, R., Dresselhaus, G., \& Dresselhaus, M. S. (2003). Characterizing carbon nanotube samples with resonance Raman scattering. New Jounal of Physics, 5, 139-1-17. http://dx.doi.org/10.1088/1367-2630/5/1/139

Han, S. P., \& Goddard, III. W. A. (2009). Coupling of Raman radial breathing modes in double-wall carbon nanotubes and bundles of nanotubes. The Journal of Physical Chemistry B, 113, 7199-7204. http://dx.doi.org/10.1021/jp805828g

Hasan, T., Sun, Z., Wang, F., Bonaccorso, F., Tan, P. H., Rozhin, A. G., \& Ferrari, A. C. (2009). Nanotube-polymer composites for ultrafast photonics. Advanced Materials, 21, 3874-3899. http://dx.doi.org/10.1002/adma.200901122

He, X. Q., Kitipornchai, S., \& Liew, K. M. (2005). Buckling analysis of multi-walled carbon nanotubes: A continuum model accounting for van der Waals interaction. Jouranl of the Mechanics and Physics of Solids. 53, 303-326. http://dx.doi.org/10.1016/j.jmps.2004.08.003

Hu, Y. G., Liew, K. M., Wang, Q., He, X. Q., \& Yakobson, B. I. (2008). Nonlocal shell model for elastic wave propagation in single- and double-walled carbon nanotubes. Jouranl of the Mechanics and Physics of Solids, 56, 3475-3485. http://dx.doi.org/10.1016/j.jmps.2008.08.010

Kurti, J., Kresse, G., \& Kuzmany, H. (1998). First-principles calculations of the radial breathing mode of $\begin{array}{lllll}\text { single-wall carbon nanotubes. Physical Review } B, \quad 58, & \text { R8869-8872. }\end{array}$ http://dx.doi.org/10.1103/PhysRevB.58.R8869

Kuzmany, H., Plank, W., Pfeiffer, R., \& Simon, F. (2008). Raman scattering from double-walled carbon nanotubes. Journal of Raman Spectroscopy, 39, 134-140. http://dx.doi.org/10.1002/jrs.1824

Lawler, H., Areshkin, D., Mintmire, J., \& White, C. (2003). Radial-breathing mode frequencies for single-walled carbon nanotubes of arbitrary chirality: First-principles calculations. Physical Review B, 72, 233403-1-4. http://dx.doi.org/10.1103/PhysRevB.72.233403

Liu, K., Wang, W., Wu, M., Xiao, F., Hong, X., Aloni, S., Bai, X., Wang, E., \& Wang, F. (2011). Intrinsic radial breathing oscillation in suspended single-walled carbon nanotubes. Physical Review B, 83, 113404-1-4. http://dx.doi.org/10.1103/PhysRevB.83.113404

Mahan, G. D. (2002). Oscillations of a thin hollow cylinder: Carbon nanotubes. Physical Review B, 65, 235402-1-7. http://dx.doi.org/10.1103/PhysRevB.65.235402

Mylvaganam, K., \& Zhang, L. C. (2004) Important issues in a molecular dynamics simulation for characterising the mechanical properties of carbon nanotubes. Carbon, 42, 2025-2032. http://dx.doi.org/10.1016/j.carbon.2004.04.004

Natsuki, T., Ni, Q. Q., \& Endo, M. (2008). Analysis of the vibration characteristics of double-walled carbon nanotubes. Carbon, 46, 1570-1573. http://dx.doi.org/10.1016/j.carbon.2008.06.058

Natsuki, T., Tantrakarn, K., \& Endo, M. (2004). Prediction of elastic properties for single- walled carbon nanotubes. Carbon, 42, 39-45. http://dx.doi.org/10.1016/j.carbon.2003.09.011

Popov, V.N., Doren, V. E.V., Balkanski, M. (1999). Lattice dynamics of single-walled carbon nanotubes. Physical Review B, 59, 355-8358. http://dx.doi.org/10.1103/PhysRevB.59.8355

Popov, V. N., \& Henrard, L. (2002). Breathinglike phonon modes of multiwalled carbon nanotubes. Physical Review B, 65, 235415-1-6. http://dx.doi.org/10.1103/PhysRevB.65.235415

Qian, D., Wagner, G. J., Liu, W. K., Yu, M. F., \& Ruoff, R. S. (2002). Mechanics of carbon nanotubes. Applied Mechanics Reviews. 55, 495-533. http://dx.doi.org/10.1115/1.1490129 
Saito, R., Matsuo, R., Kimura, T., Dresselhaus, G., \& Dresselhaus, M. S. (2001) Anomalous potential barrier of double-wall carbon nanotube. Chemical Physics Letters, 384, 187-193. http://dx.doi.org/10.1016/S0009-2614(01)01127-7

Shi, M. X., Li, Q. M., \& Huang, Y. (2009). Internal resonance of vibrational modes in single-walled carbon nanotubes. Proceedings of the Royal Society A, 465, 3069-3082. http://dx.doi.org/10.1098/rspa.2009.0147

Soedel, W. (2004). Vibration of shells and plates (3rd ed.). New York: Marcel dekker, Inc.

Srivastava, D., Menon, M., \& Cho, K. (1999). Nanoplasticity of single-wall carbon nanotubes under uniaxial compression. Physical Review Letters, 83, 2973-2976. http://dx.doi.org/10.1103/PhysRevLett.83.2973

Venkateswaran, U. D., Masica, D. L., Sumanasekera, G. U., Furtado, C. A., Kim, U. J., \& Eklund, P. C. (2003). Diameter dependent wall deformations during the compression of a carbon nanotube bundle. Physical Review B, 68, 241406-1-4. http://dx.doi.org/10.1103/PhysRevB.68.241406

Wang, C. M., Tan, V. B. C., \& Zhang, Y. Y. (2006). Timoshenko beam model for vibration analysis of multi-walled carbon nanotubes. Journal of Sound and Vibration, 294, 1060-1072. http://dx.doi.org/10.1016/j.jsv.2006.01.005

Wang, C. Y., Ru, C. Q., \& Mioduchowski, A. (2005). Pressure effect on radial breathing modes of multiwall carbon nanotubes. Journal of Applied Physics, 97, 024310-1-9. http://dx.doi.org/10.1063/1.1836007

Wu, G., Zhou, J., \& Dong, J. (2007). Radial-breathing-like phonon modes of double- walled carbon nanotubes. Physical Review B, 72, 115418-1-5. http://dx.doi.org/10.1103/PhysRevB.72.115418

Yakobson, B. I. (1998). Mechanical relaxation and intramolecular plasticity in carbon nanotubes. Applied Physics Letters, 72, 918-920. http://dx.doi.org/10.1063/1.120873

Yang, H. K., \& Wang, X. (2007). Torsional buckling of multi-wall carbon nanotubes embedded in an elastic medium. Composite Structures, 77, 182-192. http://dx.doi.org/10.1016/j.compstruct.2005.06.013

Zhang, P., Lammert, P. E., \& Crespi, V. H. (1998). Plastic deformations of carbon nanotubes. Physical Review Letters, 81, 5346-5349. http://dx.doi.org/10.1103/PhysRevLett.81.5346

\section{Copyrights}

Copyright for this article is retained by the author(s), with first publication rights granted to the journal.

This is an open-access article distributed under the terms and conditions of the Creative Commons Attribution license (http://creativecommons.org/licenses/by/3.0/). 